Supporting Information to

\title{
Bacterial production of Barley Stripe Mosaic Virus Biotemplates for Palladium Nanoparticle Growth
}

Yu-Hsuan Lee ${ }^{a} \star$, Kok Zhi Lee ${ }^{b, d}$, Rachel G. Susler ${ }^{a}$, Corren A. Scott ${ }^{a}$, Longfei Wang $^{c}$, L. Sue Loesch-Fries $^{c}$, Michael T. Harris ${ }^{a}$, Kevin V. Solomon ${ }^{b, d, e^{*}}$

a 480 Stadium Mall Drive, School of Chemical Engineering, Purdue University, West Lafayette, IN 47907, United States

${ }^{b} 225$ South University Street, Agricultural \& Biological Engineering, Purdue University, West Lafayette, IN 47907-2093, United States

c 915 West State Street, Department of Botany and Plant Pathology, Purdue University, West Lafayette, IN 47907, United States

${ }^{d} 1203$ West State Street, Bindley Bioscience Center, Purdue University, West Lafayette, IN 47906, United States

e 500 Central Drive, Laboratory of Renewable Resources Engineering (LORRE), Purdue University, West Lafayette, IN, 47907-2022, United States

$\ddagger$ equal contribution

*corresponding author. kvs@purdue.edu 
DNA sequence of BSMV-CP-linker-OAS

BSMV capsid coding sequence; linker sequence ; OAS sequence

CATATGATGCCCAACGTATCACTGACAGCGAAAGGGGGAGGTCATTACATCGAAGATCAGTGGGA TACGCAAGTCGTCGAAGCAGGAGTGTTCGACGACTGGTGGGTGCATGTAGAGGCCTGGAATAAGT TTCTTGACAATCTGCGCGGCATCAATTTTTCCGTCGCCAGCAGTCGCTCACAAGTAGCAGAGTATT TGGCTGCTTTGGATCGTGACCTTCCGGCTGACGTTGATCGTCGTTTCGCGGGTGCACGTGGTCAAA TCGGCAGCCCCAATTACTTACCAGCACCTAAATTTTTTCGTCTTGATAAACGTACAATCGCTGAATT GACACGTTTGTCGCGCTTGACGGATCAGCCCCACAACAATCGTGATATCGAGTTAAATCGCGCAA AACGCGCAACAACAAATCCTAGCCCACCTGCTCAAGCCCCGAGCGAAAACCTTACACTGCGCGAC GTGCAACCCTTAAAGGACTCCGCGTTACATTATCAGTATGTCCTTATTGATCTTCAGTCCGCACGCT TGCCTGTGTATACCCGCAAGACTTTCGAGCGCGAGCTGGCTCTGGAGTGGATCATTCCAGATGCA GAGGAAGCATAAACCGGTTTTAAATATGTCTTACAGTATCACTACTCCATCTCAGTTCGTGTTCTTG TCATCAGCGTGGGCCGACCCAATAGAGTTAATTAATTTATGTACTAATGCCTTAGGAAATCAGTTTC AAACACAACAAGCTCGAACTGTCGTTCAAAGACAATTCAGTGAGGTGTGGAAACCTTCACCACAA GTAACTGTTAGGTTCCCTGACAGTGACTTTAAGGTGTACAGGTACAATGCGGTATTAGACCCGCTA GTCACAGCACTGTTAGGTGCATTCGACACTAGAAATAGAATAATAGAAGTTGAAAATCAGGCGAA CCCCACGACTGCCGAAACGTTAGATGCTACTCGTAGAGTAGACGACGCAACGGTGGCCATAAGG AGCGCGATAAATAATTTAATAGTAGAATTGATCAGAGGAACCGGATCTTATAATCGGAGCTCTTTC GAGAGCTCTTCTGGTTTGGTTTGGACCTCTGGTCCTGCAACTTGATAGTCCGGACCTGCAGGACGC GTGTCGACGTTTGAGAGAGAAGATTACAAACGTGAGAGACGGAGGGCCCATGGAACTTACAGAA GAAGTCGTTGATGAGTTCATGGAAGATGTCCCTATGTCGATCAGGCTTGCAAAGTTTCGATCTCGA ACCGGCCTAGGCTCGTGGTATTGTTTATAGAAATAATATAAAATTAGGTTTGAGAGAGAAGATTAC AAACGTGAGAGACGGAGGGCCCATGGAACTTACAGAAGAAGTCGTTGATGAGTTCATGGAAGAT GTCCCTATGTCGATCAGGCTTGCAAAGTTTCGATCTCGAACCGGAAAAAAGAGTGATGTCCGCAA AGGGAAAAATACTCGAG 
A) Uncoated BSMV VLPs
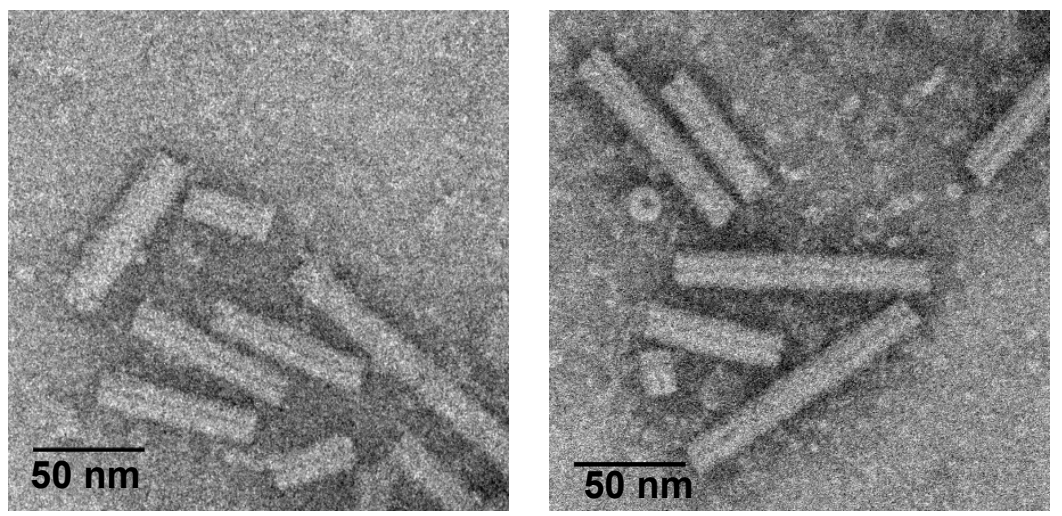

B) Pd-coated BSMV VLPs
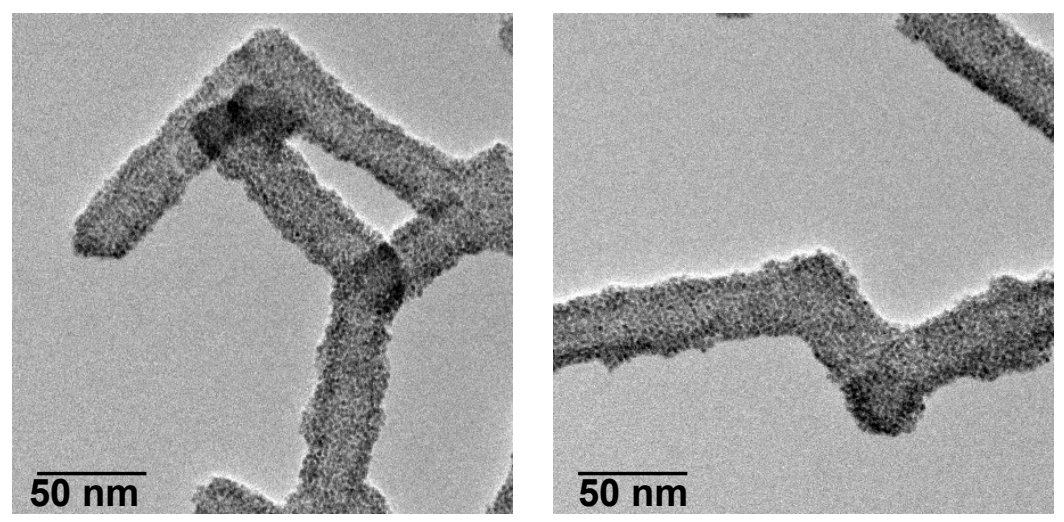

Figure S1. Transmission electron microscope (TEM) images of A) uncoated BSMV-CP-OAS VLPs and B) Pd-coated BSMV-CP-OAS VLPs. Scale bar: $50 \mathrm{~nm}$. 